\title{
Optimization of Quantum Pumps
}

Elina Potanina

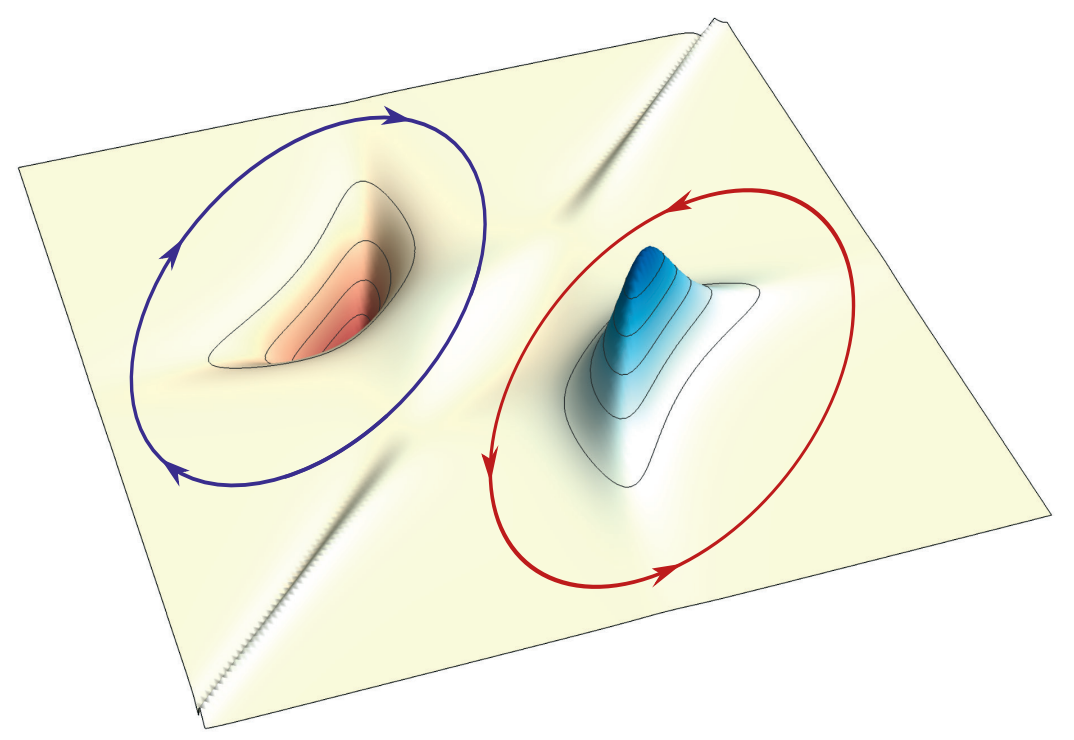




\title{
Optimization of Quantum Pumps
}

\author{
Elina Potanina
}

A doctoral dissertation completed for the degree of Doctor of Science (Technology) to be defended, with the permission of the Aalto University School of Science, at a public examination held at the lecture hall M1 of the school on 17th of January 2020 at 13:00. 
Professor Christian Flindt, Aalto University, Finland

\section{Preliminary examiners}

Professor emerita Ora Entin-WohIman, Tel Aviv University, Israel

Dr. Rafael Sanchez, Autonomous University of Madrid, Spain

\section{Opponent}

Professor Peter Samuelsson, Lund University, Sweden

DOCTORAL DISSERTATIONS 234/2019

(C) 2019 Elina Potanina

ISBN 978-952-60-8880-8 (printed)

ISBN 978-952-60-8881-5 (pdf)

ISSN 1799-4934 (printed)

ISSN 1799-4942 (pdf)

http://urn.fi/URN:ISBN:978-952-60-8881-5

Unigrafia Oy

Helsinki 2019

Finland 
Author

Elina Potanina

Name of the doctoral dissertation

Optimization of Quantum Pumps

\begin{tabular}{l}
\hline Publisher School of Science \\
\hline Unit Department of Applied Physics \\
\hline Series Aalto University publication series DOCTORAL DISSERTATIONS 234/2019 \\
\begin{tabular}{lll}
\hline Field of research Theoretical condensed matter physics & Date of the defence 17 January 2020 \\
\hline Manuscript submitted 12 September 2019 & Language English \\
\hline Permission for public defence granted (date) & 11 November 2019 \\
\hline$\square$ Monograph & $\square$ Article dissertation & Essay dissertation
\end{tabular}
\end{tabular}

\section{Abstract}

The SI unit system has recently moved away from artificial definitions of units to the elegant quantum definitions based on natural constants. The previous definition of the ampere involved the force between two infinitely long wires, and it is now replaced by the quantum ampere, where current is defined using the elementary charge and caesium frequency standard. Recent developments in quantum technology and nano-device fabrication have already enabled ondemand single-electron delivery. Experimental realization of the quantum ampere with close-tometrological accuracy was recently demonstrated using single-electron pumps based on quantum dots with tunable-barriers.

In this thesis, I develop optimization schemes tailored for the experimentally available devices such as single-electron turnstiles and tunable-barrier quantum pumps. I employ theories of quantum transport for periodically driven systems in the low- and high-frequency regimes, to answer the following questions: What is the optimal operation cycle for a quantum pump to achieve high accuracy in the $\mathrm{GHz}$ regime? How can we increase the breakdown frequency of single-electron pumps?

I optimize the regularity of emitted electrons in a turnstile using the distribution of electron waiting-times. I provide an analytic optimization of two-parameter charge pumps based on the symmetries of the corresponding Berry curvature. For one-parameter pumps, I evaluate the breakdown frequency via a high-frequency expansion and optimize it so that it increases by one order of magnitude. Within the framework of non-equilibrium quantum thermodynamics, I demonstrate how it is possible to maximize the coefficient of performance for coherent pumps.

Keywords Nanoelectronics, quantum pumps, adiabatic pumping, single-electron pumps, counting statistics, distribution of waiting times

\begin{tabular}{lc}
\hline ISBN (printed) $978-952-60-8880-8$ & ISBN (pdf) $978-952-60-8881-5$ \\
\hline ISSN (printed) $1799-4934$ & ISSN (pdf) $1799-4942$ \\
\hline Location of publisher Helsinki & Location of printing Helsinki Year 2019 \\
\hline Pages 86 & urn http://urn.fi/URN:ISBN:978-952-60-8881-5 \\
\hline
\end{tabular}





\section{Preface}

The work presented in this thesis was carried out in the Quantum Transport group, Department of Applied Physics, Aalto University during the years 2016-2019.

I joined the Quantum Transport group at the beginning of 2016. Besides the group leader Prof. Christian Flindt, it consisted of postdoctoral researchers only. Thank you, Christian, for introducing me to the waiting time theory and full counting statistics. Thank you, for providing full research freedom to me. Thank you, Shuo, for always being a friendly and supportive postdoc.

The first person to offer me help with documents and the Finnish language was Joel Röntynen. At that time, Joel was a $\mathrm{PhD}$ student in Teemu Ojanen's group. I would like to express my gratitude to them. Thank you, Joel, for your support and help, for going through the tough times together, and for the postcards! Thank you, Teemu, for consulting me on topological pumping and making me feel confident about the knowledge I have. Also, I would like to thank two former PhD students of Teemu, Kim Pöyhönen and Alex Westström for helping me to apply for doctoral studies and providing all the necessary information.

At some point during the first year, two more $\mathrm{PhD}$ students joined the group. Thank you, Aydin, for going through the struggles of doctoral studies together and for the fun times at the conferences! Thank you, Paul, for bearing with my sometimes-too-much talking and for your contribution to my knowledge of German language, "Ich habe eine adiabatische matrix zu konstruieren!".

After one and a half years, I had my midterm and Jukka Pekola was my opponent. Thank you, Jukka, for a kind and constructive feedback on my midterm report and presentation!

By the end of the second year, one more postdoc joined the group. Thank 
you, Pablo, for always being nice and patient with me, and thank you for proofreading my thesis! Soon after Pablo joined, first Finnish members joined the group - Janne, Tuomas, and Tony. All three were bachelor students at that time and I learned a lot about Aalto University from an undergraduate perspective. Thank you, guys! Many special thanks go to Tony for being a reliable great friend and introducing me to the great coffee world!

Each year Christian invited Misha Moskalets from Kharkiv Polytechnic Institute, Ukraine, to come for three months as a visitor to our group. Thank you, Misha, for introducing Floquet matrices to me, sharing your knowledge, and your support! And Thank you, Christian, for inviting Misha!

We also had Fredrik Brange as a visitor for a couple of months and now he has joined the group as a postdoc. I have enjoyed your company in the office quite a lot, Fredrik! Thank you!

Besides theoretical research, I had a pleasure to collaborate with a talented experimentalist from Mikko Möttönen's group Máté Jenei. Thank you, Máté, for teaching me all the subtleties of experimental physics! Thank you, Mikko, for your constructive criticism!

While I didn't experience the lack of ideas, I certainly suffered from the absence of a frequent, meaningful and deep human interaction in the academic environment. It is impossible to measure the level of support and encouragement coming from Prof. Slava Kashcheyevs, my former supervisor. Thank you, Slava, for the infinite flow of positive energy. From Slava I learned that physics is a social science. My doctoral studies only confirmed this statement, as my ideas and scientific productivity grew exponentially in the presence of sincere, open and emotionally deep communication.

Towards the end of my Ph.D., in summer 2019, more collaborations with experimental physics groups occurred. I would like to thank Prof. Rolf Haug for inviting me to a workshop in Hannover and sharing the experimental results of his group with me! Special thanks to Johannes Bayer and Adrian Schmidt, students of Prof. Haug, for the fruitful discussions we had during the workshop. I would also like to thank Timo Wagner, whom I met during the DPG meeting in Berlin in 2017. Thank you, Timo, for enlightening discussions on waiting-time distributions!

At the same workshop in Hannover, as a coincidence (or not?) I met Slava once again. Our discussions resulted in a collaboration with Prof. Giuseppe 
Tettamanzi from the university of Adelaide. Thank you, Giuseppe, for sharing the two-atom pumping results with me and explaining all the details of the experiment! I was extremely happy to hear from Giuseppe about the adiabatic pumping measurements, while Slava and I recognized the Berry curvature imprints in these measurements.

Last but not least, a gigantic acknowledgement goes to my family and friends. Thanks to my dad, Vladimir, a mechanical engineer, who taught me how a car engine works when I was five. It definitely was a great beginning of my physics studies. I will always be in debt to my mom, Olga, for the enormous amount of love and support. Thank you, mom, for being the greatest example of a truly amazing woman! Thanks to my grandmother, Liubov, a hydro-technical engineer, who helped me solving the hydrodynamics problems and gave me all the care and love she could. Special thanks to our family friend Eugene, his visits were always as great as a celebration of the new year and a birthday combined!

Thanks to my university friends with whom we studied together - Lāsma, Krista and Reinis, for regularly sharing all the after-graduation experiences with me and their support. Thank you, Agnese, for being a great friend and regularly sending me the quantum coherent postcards! Thanks to my true engineer friend Ksenija, who is a master of really applied technologies and a doctor of really good friendships! Thank you, Vita, for your cute and supportive postcards, and educating me in ornithology! I am blessed to have such a sensitive and understanding friend like you, Vita. Big thanks to the amazing physicists Lara Ulčakar and Jemma Needham for their positive attitude, support and heart-warming postcards! Special thanks to exceptionally talented AScI interns, Alexandra Mestre and Aaron Altman! It was a pleasure to supervise you.

Helsinki, December 3, 2019,

Elina Potanina 
Preface 


\section{Contents}

$\begin{array}{ll}\text { Preface } & 1\end{array}$

$\begin{array}{ll}\text { Contents } & 5\end{array}$

$\begin{array}{ll}\text { List of Publications } & 7\end{array}$

$\begin{array}{ll}\text { Author's Contribution } & 9\end{array}$

$\begin{array}{ll}\text { 1. Introduction } & 11\end{array}$

2. Single-electron pumping 13

2.1 Coulomb blockade and rate equations . . . . . . . . . 13

2.2 Full counting statistics . . . . . . . . . . . 16

2.2.1 Electronic waiting times . . . . . . . . 16

2.2.2 Statistics and Floquet eigenvalues . . . . . 20

2.3 Adiabatic pumping and classical Berry phase . . . . . . 21

2.4 Non-adiabatic pumping . . . . . . . . . . . . . . 23

3. Coherent single-electron transport 27

3.1 Floquet scattering theory . . . . . . . . . . . 27

3.2 Thermodynamics of coherent driven conductors . . . . . 30

3.3 Adiabatic-linear response and Berry phase . . . . . . . . . 31

4. Summary of the findings 35

$\begin{array}{ll}\text { References } & 37\end{array}$

$\begin{array}{ll}\text { Publications } & 41\end{array}$ 


\section{List of Publications}

This thesis consists of an overview and of the following publications which are referred to in the text by their Roman numerals.

I Elina Potanina and Christian Flindt. Electron waiting times of a periodically driven single-electron turnstile. Physical Review B, 96, 045420, July 2017.

II Máté Jenei, Elina Potanina, Ruichen Zhao, Kuan Y. Tan, Alessandro Rossi, Tuomo Tanttu, Kok W. Chan, Vasilii Sevriuk, Mikko Möttönen, and Andrew Dzurak. Waiting time distributions in a two-level fluctuator coupled to a superconducting charge detector. Accepted, in production in Physical Review Research, arXiv:1909.02866 , November 2019.

III Elina Potanina, Kay Brandner, and Christian Flindt. Optimization of quantized charge pumping using full counting statistics. Physical Review B, 99, 035437, January 2019.

IV Elina Potanina, Christian Flindt, Michael Moskalets, and Kay Brandner. Thermodynamic bounds on coherent transport in periodically driven conductors. Submitted to Physical Review X, arXiv:1906.04297, June 2019 . 
List of Publications 


\section{Author's Contribution}

\section{Publication I: "Electron waiting times of a periodically driven single-electron turnstile"}

The author performed analytic derivations and the numerical calculations, contributed to the preparation of the manuscript, and made all the figures.

\section{Publication II: "Waiting time distributions in a two-level fluctuator coupled to a superconducting charge detector"}

The author formulated the theory of waiting times for a particular experimental setup and wrote half of the manuscript including abstract, introduction, conclusions and the waiting-time theory section.

\section{Publication III: "Optimization of quantized charge pumping using full counting statistics"}

The author formulated the original problem and came up with a solution to it, carried out analytic derivations and numerical simulations, contributed to the preparation of the manuscript, and made all the figures.

\section{Publication IV: "Thermodynamic bounds on coherent transport in periodically driven conductors"}

The author came up with an idea to study the thermodynamic cost of coherent single-electron pumping, did analytic derivations of the adiabatic coefficients and numerical calculations to illustrate the bounds, made all the figures and contributed to the preparation of the manuscript. 
Author's Contribution 


\section{Introduction}

On May 20, 2019, we have finally moved from the artificial definition of the ampere involving the force between two infinitely long wires with a negligible circular cross-section to an elegant quantum ampere. The new ampere is defined using the elementary charge $e$ and the caesium frequency standard $\Delta \nu_{C s}$. One of the officially approved experimental realizations of the new ampere is single-electron pumping through a tunable-barrier quantum dot [1, 2] via periodic driving with frequency $f$ such that the current is given by the number of transferred electrons $n$ per cycle:

$$
I=n e f
$$

Recent developments in quantum technology and nano-device fabrication have already enabled single-electron pumping with close-to-metrological accuracy [3, 4, 5]. Dynamic single-electron sources are also of practical importance as periodic emission of single electrons is crucial for synchronized many particle operations in quantum information processors [6]. Dynamic single-electron transistors are building blocks for logic operations [7] and sensitive read-out devices [8] for a solid-state quantum computer.

This thesis focuses on the optimization of periodically driven devices in the low- and high-frequency regimes. For example, how should we operate a nano-device in order to get a perfectly quantized current in the output? What is the optimal operation cycle for a quantum pump to achieve maximal efficiency? There is no universal solution of such inverse time-dependent problems. In order to answer these questions, we develop the optimization schemes tailored for the experimentally available and relevant devices such as single-electron turnstiles and tunable-barrier quantum pumps.

Publication I demonstrates that the distribution of waiting times between subsequent tunneling events in a single-electron turnstile reveals the regularity of electron emission events. Publication II is a blend of experiment 
and theory, where we directly extract the time scales of electronic transitions in a charge trap via distributions of waiting times. Publication III contains an optimization scheme for single- and two-parameter quantum pumps. Finally, in Publication IV, we derive and illustrate a power-flux trade-off relation for coherent single-electron pumps using the geometric optimization argument.

The structure of this overview is the following. Chapter 2 briefly discusses the concept of single-electron pumping and statistics of charge transfer in the Coulomb blockade regime. Chapter 3 contains an introduction to coherent transport, Floquet scattering theory, and non-equilibrium thermodynamics of mesoscopic conductors. Finally, Chapter 4 gives a summary of our key findings. 


\section{Single-electron pumping}

In this chapter, we introduce the concept of single-electron pumping in the presence of Coulomb blockade and the counting statistics of tunneling events. Later, we discuss distributions of waiting times between singleelectron emission events. The end of this chapter focuses on two different pump operation regimes usually referred to as adiabatic and non-adiabatic pumping.

\subsection{Coulomb blockade and rate equations}

Single-electron pumps based on a metallic island with tunable-barriers are subjects to the Coulomb blockade. A metallic island is a nano-scale object with size less than $L=100 \mathrm{~nm}$ in all three dimensions. From the size of the island, we can immediately estimate the order of Coulomb interactions as $e^{2} / L \sim 10^{-3} \mathrm{eV}$. It sets the largest energy scale - the charging energy $E_{C}$, which tells us how much energy is needed to charge an island by adding or removing one electron. The charge of the island, $Q=e n$ generates an electric field and accumulates electrostatic energy given by:

$$
E=\frac{e^{2} n^{2}}{2 C}=E_{C} n^{2}
$$

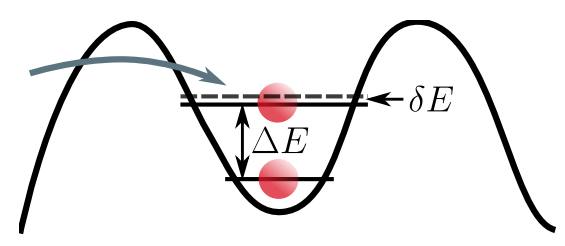

Figure 2.1. A metallic island formed by electrostatically induced potential barriers. The energetic cost of adding one electron to the island is equal to the electrostatic energy change $\Delta E$, while the mean level spacing between electron states can be ignored $(\delta E \ll \Delta E)$ in the regime of Coulomb blockade. 
where $C$ is the capacitance of the metallic island. The amount of energy needed to add one electron to the island is given by the change in electrostatic energy:

$$
\Delta E=E_{C}(n+1)^{2}-E_{C} n^{2}=E_{C}(2 n+1) .
$$

Electrostatic energy is a purely classical concept. There is also a quantum energy scale related to the discrete electron states - the mean level spacing $\delta E$. Given that every atom has at least one valence electron, there is around $N_{a}=10^{9}$ electrons in the island of a volume $L^{3}$. The mean levelspacing $\delta E$ is inversely proportional to $N_{a}$ and therefore becomes irrelevant $(\delta E \ll \Delta E)$ as depicted in Fig. 2.1.

Single-electron pumping is a dynamic process composed of tunneling events. Tunneling is a quantum process, but presence of the Coulomb blockade leads to vanishing coherence between the charge states. An equilibrium charge state of the metallic island is certain at any time. Therefore, the charge dynamics of the island can be described by a rate equation:

$$
\frac{d}{d t}|P(t)\rangle=\mathbf{L}(t)|P(t)\rangle
$$

where the vector $|P(t)\rangle=\left[p_{0}(t), p_{1}(t), p_{2}(t), \ldots\right]^{T}$ contains the probabilities for the island to be occupied by $0,1,2, \ldots$ electrons. The rate matrix $\mathbf{L}(t)$ describes the transitions between different charge states of the island. The "braket" notation here is used for convenience, but we note that the left and right vectors $|P(t)\rangle,\langle P(t)|$ are not related by Hermitian conjugation, since the rate matrix is not Hermitian.

Let us look at the case when the island is either empty or occupied by one electron. The rate matrix then takes the simple form

$$
\mathbf{L}(t)=\left(\begin{array}{ll}
-\Gamma_{L}^{+}(t)-\Gamma_{R}^{+}(t) & \Gamma_{L}^{-}(t)+\Gamma_{R}^{-}(t) \\
\Gamma_{L}^{+}(t)+\Gamma_{R}^{+}(t) & -\Gamma_{L}^{-}(t)-\Gamma_{R}^{-}(t)
\end{array}\right),
$$

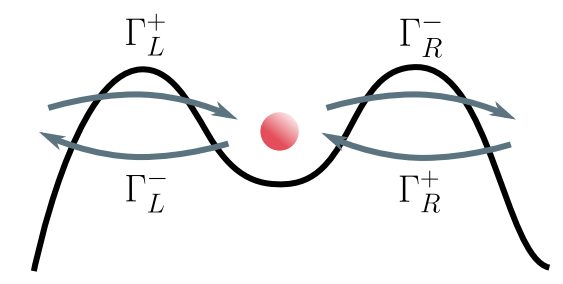

Figure 2.2. A metallic island occupied by one electron. Arrows indicate all the allowed transitions. 
where $\Gamma_{\alpha}^{ \pm}(t)$ is the rate at which tunneling occurs between the island and the leads, changing the occupation by \pm 1 electron with charge $e$, Fig. 2.2.

Sequential single-electron tunneling rates are given by the Fermi golden rule assuming weak tunnel coupling:

$$
\Gamma_{\alpha}^{ \pm}(t)=\frac{G_{\alpha}(t)}{e^{2}} \frac{ \pm \Delta E(t)}{\exp [ \pm \beta \Delta E(t)]-1},
$$

where the barrier conductances $G_{\alpha}(t)$ are functions of the gate voltages and $\beta=1 / k_{B} T$ is the inverse temperature. For example, in the silicon pumps, conductance depends exponentially on the gate voltages $G_{\alpha}(t)$ as $G_{\alpha}(t)=G_{\alpha} \exp \left[V_{\alpha}(t) / V_{s}\right]$, where $V_{s}$ is known as the sub-threshold slope [9]. The change of the electrostatic energy due to the addition of an electron reads $\Delta E(t)=-E_{\mathrm{c}}\left[\mathcal{N}_{g}+2\left\{C_{L} V_{L}(t)+C_{R} V_{R}(t)\right\} / e\right]$, where $C_{L}$ and $C_{R}$ are the gate-island capacitances, $E_{\mathrm{c}}=e^{2} / 2\left(C_{L}+C_{R}+C_{S}+C_{D}\right)$ is the charging energy with source-dot and dot-drain capacitances $C_{S}$ and $C_{D}$, and $\mathcal{N}_{g}$ is the offset, see Fig. 2.3.

Higher-order processes like co-tunneling are possible but happen to be suppressed for two reasons. The co-tunneling rate $\Gamma_{c o}$ can be estimated from the rate product and the conductance ratio [10] $\Gamma_{c o} \sim \Gamma_{L}^{+} \Gamma_{R}^{-} G / G_{Q}$, where $G_{Q}$ is the conductance quantum. Generally, during the pumping cycle, the product of the source-to-island and island-to-drain tunneling rates is kept small to avoid leakage of charge in the wrong direction. In the first half of the cycle, source-to-island rate dominates $\Gamma_{L}^{+}(t) \gg$ $\Gamma_{R}^{-}(t)$ and for the second half of the cycle the inequality is reversed. The barrier conductances are kept below the value of the conductance quantum $G_{L, R}(t)<G_{Q}$, in order to ensure the regime of Coulomb blockade. At all times $t$, co-tunneling can be safely ignored as $G / G_{Q}<1$ and $\Gamma_{L}^{+} \Gamma_{R}^{-} \rightarrow 0$.

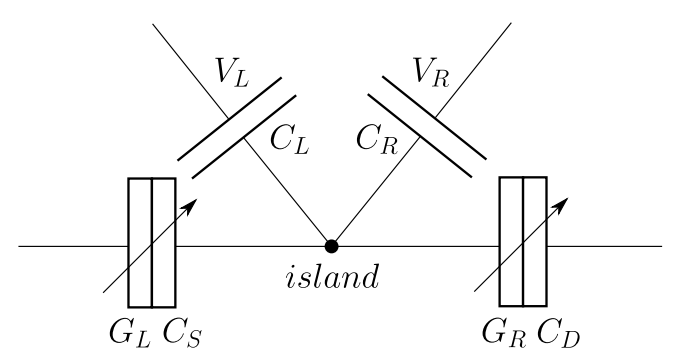

Figure 2.3. A metallic island separated from the source and drain by electrostatically induced barriers with conductances $G_{L / R}$, and source-dot/dot-drain capacitances $C_{S / D}$. The tunnel barriers are formed by applying voltages $V_{L / R}$ on corresponding gates, where $C_{L / R}$ are the gate-island capacitances. 


\subsection{Full counting statistics}

The statistics of tunneling events is encoded in the probability $P(n, t)$ of $n$ electrons being transferred to the collector during the time-span $[0, t]$. This probability evolves according to the rate equation

$$
\frac{d}{d t}|P(n, t)\rangle=\mathbf{L}_{0}(t)|P(n, t)\rangle+\mathbf{J}_{+}(t)|P(n-1, t)\rangle+\mathbf{J}_{-}(t)|P(n+1, t)\rangle,
$$

where rate matrix is partitioned as $\mathbf{L}(t)=\mathbf{L}_{0}(t)+\mathbf{J}_{+}(t)+\mathbf{J}_{-}(t)$ with jump operator $\mathbf{J}_{ \pm}(t)$ describing charge transfers or "jumps" to and from the collector. Rate equations become decoupled by introducing the counting field $\chi$ via the definition $|P(\chi, t)\rangle \equiv \sum_{n}|P(n, t)\rangle e^{i n \chi}$. We then arrive at the modified master equation

$$
\frac{d}{d t}|P(\chi, t)\rangle=\mathbf{L}(\chi, t)|P(\chi, t)\rangle .
$$

The solution of the modified master equation is a moment generating function $\mathcal{M}(\chi, t) \equiv \sum_{n} P(n, t) e^{i n \chi}=\langle 1 \mid P(\chi, t)\rangle$, which provides us with the moments of $n$ :

$$
\left\langle n^{m}\right\rangle(t)=\left.\partial_{i \chi}^{m} \mathcal{M}(\chi, t)\right|_{\chi=0} .
$$

Generally, we are interested not only in the mean number of pumped particles but in the noise as well. The noise is given by a second cumulant $\left\langle n^{2}\right\rangle-\langle n\rangle^{2}=\left\langle\left\langle n^{2}\right\rangle\right\rangle$. Cumulants follow similarly from a cumulant generating function $\mathcal{S}(\chi, t) \equiv \ln \mathcal{M}(\chi, t)$ :

$$
\left\langle\left\langle n^{m}\right\rangle\right\rangle(t)=\left.\partial_{i \chi}^{m} \mathcal{S}(\chi, t)\right|_{\chi=0} .
$$

Let us now focus on the solution of the modified rate equation (2.1). Formally, the solution $|P(\chi, t)\rangle=\mathbf{U}(\chi, t)|P(\chi, 0)\rangle$ is given by the time-ordered exponential $\mathbf{U}(\chi, t)=\hat{T}\left\{e^{\int_{0}^{t} d t^{\prime} \mathbf{L}\left(\chi, t^{\prime}\right)}\right\}$. In practice, even the simplest examples can be rarely solved analytically. Ideally, a single-electron pump should deliver one electron per cycle, such that electron emission events would be separated by one period on the timeline which brings us to the concept of waiting times, see Fig. 2.4. It turns out that in order to find the distribution of waiting times between emission events, we only need to know the probability of having no such events.

\subsubsection{Electronic waiting times}

Assume there was an emission event at time $t_{e}$. The next such event will occur after the waiting time $\tau$. We pick a random time $t_{0}$ to sample the timeline (Fig. 2.5) between $t_{e}$ and $t_{0}+\tau$, and find the probability of the 

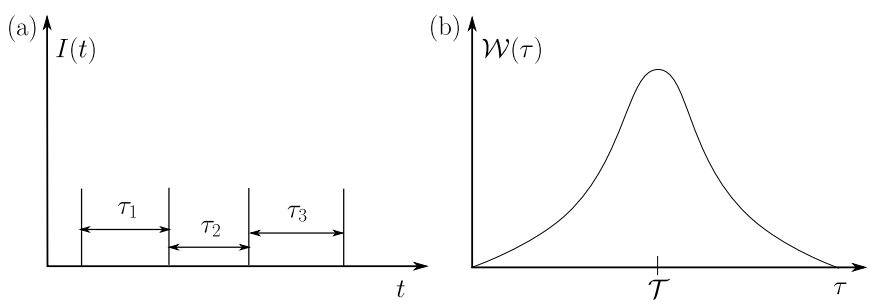

Figure 2.4. (a) Waiting times between detected events. (b) Distribution of waiting times peaked around the period of the drive when emission events occur regularly.

next event not occurring. Integrating the waiting-time distribution (WTD) $\mathcal{W}\left(t-t_{e}\right)$ over the given time span yields the probability of having emission events, so the chance of having no events is:

$$
\tilde{\Pi}\left(t_{0}+\tau, t_{e}\right)=1-\int_{t_{e}}^{t_{0}+\tau} d t \mathcal{W}\left(t-t_{e}\right) .
$$

We integrate the "no-event" probability (2.2) over all the possible emission times prior the $t_{0}$ with a probability $w\left(t_{e}\right)$ of emission event happening exactly at time $t_{e}$ :

$$
\tilde{\Pi}\left(\tau, t_{0}\right)=\alpha \int_{-\infty}^{t_{0}} d t_{e} w\left(t_{e}\right)\left(1-\int_{t_{e}}^{t_{0}+\tau} d t \mathcal{W}\left(t-t_{e}\right)\right),
$$

where $\alpha$ is a normalization factor and the probability $w\left(t_{e}\right)$ is normalized over the period $\int_{0}^{\mathcal{T}} d t / \mathcal{T} w\left(t_{e}\right)=1$. To establish the connection with the waiting-time distribution, we use the normalization condition $\int_{0}^{t} d \tau \mathcal{W}(\tau)+$ $\int_{t}^{\infty} d \tau \mathcal{W}(\tau)=1$ and rewrite Eq. (2.3) as

$$
\begin{aligned}
& \tilde{\Pi}\left(\tau, t_{0}\right)=\alpha \int_{-\infty}^{t_{0}} d t_{e} w\left(t_{e}\right)\left(1-\int_{0}^{t_{0}+\tau-t_{e}} d\left(t-t_{e}\right) \mathcal{W}\left(t-t_{e}\right)\right) \\
& =\alpha \int_{-\infty}^{t_{0}} d t_{e} w\left(t_{e}\right) \int_{t_{0}+\tau-t_{e}}^{\infty} d\left(t-t_{e}\right) \mathcal{W}\left(t-t_{e}\right) .
\end{aligned}
$$

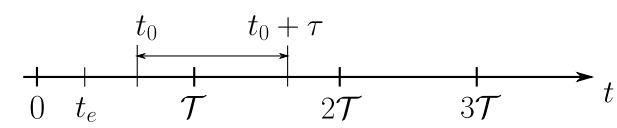

Figure 2.5. The time line depicting the period of the drive $\mathcal{T}$, the emission event time $t_{e}$, the waiting time $\tau$ and a randomly picked initial time $t_{0}$. 
Taking a double derivative with respect to the waiting time $\tau$ reveals the connection:

$$
\begin{aligned}
\partial_{\tau}^{2} \tilde{\Pi}\left(\tau, t_{0}\right) & =-\alpha \partial_{\tau} \int_{-\infty}^{t_{0}} d t_{e} w\left(t_{e}\right) \mathcal{W}\left(t_{0}+\tau-t_{e}\right) \\
& =-\alpha \partial_{\tau}\left[w\left(t_{0}\right) \int_{-\infty}^{t_{0}} d t_{e} \mathcal{W}\left(t_{0}+\tau-t_{e}\right)-\int_{-\infty}^{t_{0}} d t_{e} \mathcal{W}\left(t_{0}+\tau-t_{e}\right) \partial_{t_{e}} w\left(t_{e}\right)\right] \\
& =\alpha \partial_{\tau}\left[\int_{\infty}^{\tau} d\left(t_{0}+\tau-t_{e}\right) \mathcal{W}\left(t_{0}+\tau-t_{e}\right)-\int_{-\infty}^{t_{0}} d t_{e} \mathcal{W}\left(t_{0}+\tau-t_{e}\right) \partial_{t_{e}} w\left(t_{e}\right)\right] \\
& =\alpha\left(w\left(t_{0}\right) \mathcal{W}\left(t_{0}, \tau\right)+\partial_{\tau} \partial_{t_{0}} \tilde{\Pi}\left(\tau, t_{0}\right)\right) .
\end{aligned}
$$

The integral over the period allows us to extract the distribution of waiting times:

$$
\mathcal{W}(\tau)=\alpha^{-1} \int_{0}^{\mathcal{T}} \frac{d t_{0}}{\mathcal{T}}\left[\partial_{\tau}^{2} \tilde{\Pi}\left(\tau, t_{0}\right)-\partial_{\tau} \partial_{t_{0}} \tilde{\Pi}\left(\tau, t_{0}\right)\right]=\alpha^{-1} \partial_{\tau}^{2} \Pi(\tau)
$$

where $\Pi(\tau)$ is usually referred to as the idle-time probability - the chance of having no events during time $\tau$. The factor $\alpha^{-1}$ is the mean waiting time $\langle\tau\rangle$ which we determine from the normalization condition:

$$
\alpha^{-1} \int_{0}^{\infty} d \tau \partial_{\tau}^{2} \Pi(\tau)=1 \quad \rightarrow \quad \alpha=-\dot{\Pi}(\tau=0) .
$$

The solution of the modified equation contains all the components of the probability vector $|P(\chi, t)\rangle)=\sum_{n}|P(n, t)\rangle e^{i n \chi}$, but in fact we only need to know $P(n=0, t)$, the probability of having no electrons transferred during time $\tau$. To find the idle-time probability, we have to solve the modified rate equation (2.1) in the limit $\chi \rightarrow i \infty$ :

$$
\Pi(\tau)=\langle 1 \mid P(0, \tau)\rangle=\lim _{\chi \rightarrow i \infty}\langle 1 \mid P(\chi, \tau)\rangle .
$$

In practice, it is always easier to find the idle-time probability than to solve the full problem for all values of $\chi$. In Publication I, we show that WTDs contain a clear imprint of emitted electron regularity. For example, for a single-electron turnstile shown in Fig. 2.6, under harmonic driving $\Gamma_{L}(t)=\Gamma(\sin (2 \pi f t)+1) / 2$ and $\Gamma_{R}(t)=\Gamma(\cos (2 \pi f t)+1) / 2$, distributions of waiting times at low frequencies $\varepsilon=\Gamma / f>1$ reveal the tunneling event "leakage", Fig. 2.7. When operating in the low frequency regime, WTD develops peaks around values smaller than one period. Since the turnstile is biased and the tunneling rates are finite during the driving cycle, more than one in-and-out tunneling event occurs, causing a leakage. Instead of 
a

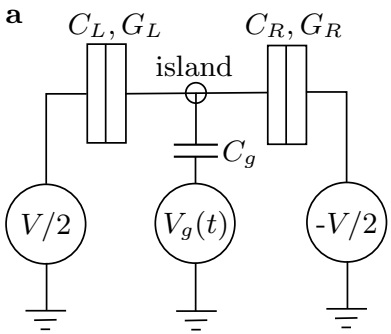

b

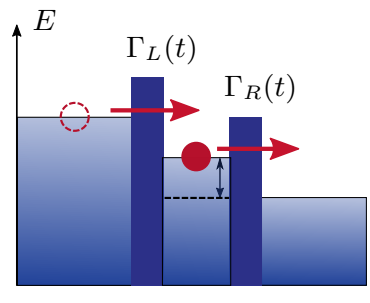

Figure 2.6. Dynamic single-electron turnstile. (a) The turnstile consists of a metallic island coupled to source and drain electrodes via two tunnel junctions with capacitances $C_{L / R}$ and tunnel conductances $G_{L / R}$. A constant voltage $V$ ensures that the transport is uni-directional at low temperatures, $\mathrm{eV} \gg k_{B} T$. A time-dependent gate voltage $V_{g}(t)$ is used to modify the transport through the island. (b) Tunneling through the tunnel junctions occurs with the timedependent rates $\Gamma_{L}(t)$ and $\Gamma_{R}(t)$ controlled by the gate voltage $V_{g}(t)$.

loading and unloading one electron, there is a stationary flow of electrons through the system and regularity of emitted electrons is absent. The WTD with finite peaks around integer multiples of period corresponds to the cycle missing events and tells us that some of the emission events are separated by 2 periods, 3 periods, etc, so regularity is lost. Cycle-missing events occur in the high-frequency regime $\epsilon<1$, when the system doesn't "have enough time" to respond to the driving.

The situation changes when the turnstile is driven according to the steplike protocol $\Gamma_{L}(t)=\Gamma \Theta(t-\lfloor t+\mathcal{T} / 2\rfloor)$ and $\Gamma_{R}(t)=\Gamma[1-\Theta(t-\lfloor t+\mathcal{T} / 2\rfloor)]$. The step-like driving assumes infinitely high barriers preventing the leakage of the unnecessary tunnelling events during the cycle. In the adiabatic regime, we observe from Fig. 2.8 a WTD sharply peaked around the period. More examples of WTDs and their detailed analysis can be found in Publication I.

For stationary transport systems, the WTD is a useful tool to directly

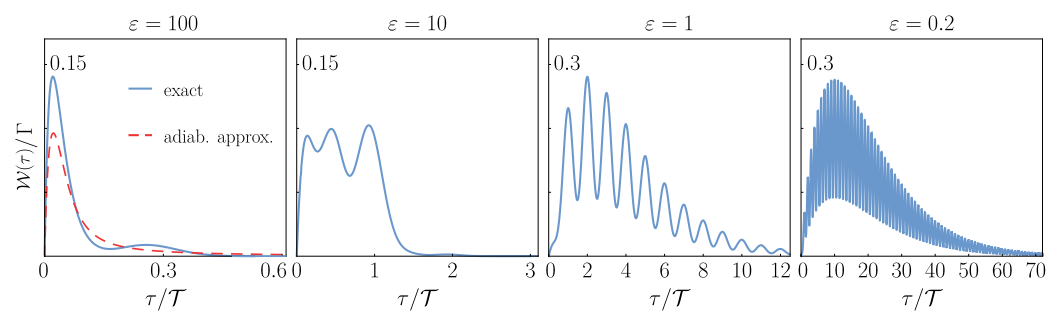

Figure 2.7. Distribution of electron waiting times for a single-electron turnstile driven by a harmonic gate voltage. We show results for the adiabatic regime, where $\varepsilon>1$ to the non-adiabatic regime, $\varepsilon<1$. In the adiabatic regime, the WTD can be approximated by an average over WTDs corresponding to stationary processes with fixed rates. 


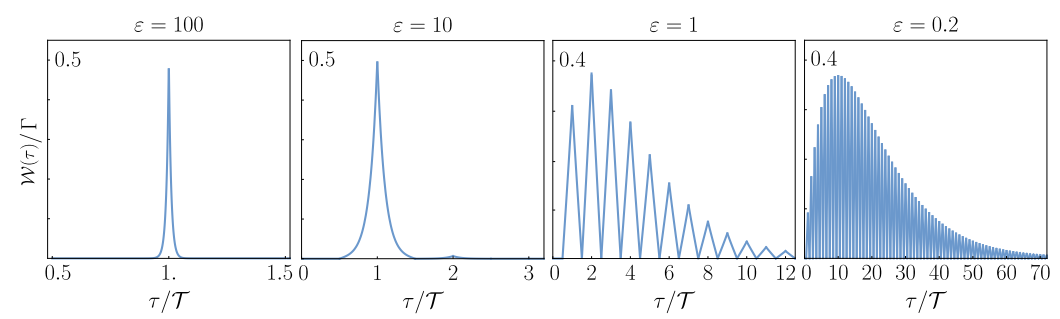

Figure 2.8. Distribution of electron waiting times for a single-electron turnstile driven by a square-wave gate voltage.

extract the time scales of electron transitions from the measured signal of the charge detector. In a recent experiment [11], waiting-time distributions were used to estimate the tunneling rates and optimize single-electron spin-readout fidelity. In Publication II, we analyze charge fluctuations in a parasitic state strongly coupled to a superconducting Josephson-junctionbased charge detector. Parasitic states including charge traps are present in almost all solid-state devices and there have been several proposals on how to avoid them [12, 13, 14, 15, 16, 17]. However, when time scales of charge fluctuations in a trap are significantly different than those of the operation of the measured device, their hindering effect can be mitigated. The charge dynamics of the parasitic state resembles that of electron transport in a quantum dot with two charge states. By constructing the distribution of waiting times from the measured detector signal and comparing it with a waiting time theory, we extract the electron in- and out-tunneling rates for the two-level fluctuator.

\subsubsection{Statistics and Floquet eigenvalues}

Besides the regularity of emitted electrons, another important aspect of single-electron pumps is the accuracy. The accuracy tells us how far the mean value of the pumped particle number is from the perfectly quantized integer value. We proceed with a discussion on the relation between Floquet eigenvalues and statistics of quantum pumps. Floquet eigenvalues turn out to be the key aspect in the analytic evaluation of the cumulants.

Dynamics and counting statistics of the quantum pump for all time-scales are encoded in the modified rate equation:

$$
\frac{d}{d t}|P(\chi, t)\rangle=\mathbf{L}(\chi, t)|P(\chi, t)\rangle .
$$

The structure of the rate equation is, in fact, very similar to the timedependent Schrödinger equation. With the main difference being that $\mathbf{L}(\chi, t)$ is a non-Hermitian operator, we can still use the idea of a Rayleigh- 
Schrödinger perturbation scheme.

Before discussing perturbation schemes for adiabatic and non-adiabatic pumping regimes, we will take advantage of $\mathbf{L}(\chi, t)=\mathbf{L}(\chi, t+\mathcal{T})$ being time-periodic and apply Floquet theorem. Solution of the rate equation in the Floquet form reads:

$$
\langle 1 \mid P(\chi, t)\rangle=\sum_{i} e^{\lambda_{i}(\chi) t}\left\langle 1 \mid p_{i}(\chi, t)\right\rangle,
$$

where $\left|p_{i}(\chi, t)\right\rangle=\left|p_{i}(\chi, t+\mathcal{T})\right\rangle$ solves the Floquet eigenvalue problem:

$$
\left[\mathbf{L}(\chi, t)-\frac{d}{d t}\right]\left|p_{i}(\chi, t)\right\rangle=\lambda_{i}(\chi)\left|p_{i}(\chi, t)\right\rangle .
$$

From Eq. (2.4), we see that the solution at long times $t$ is dominated by the eigenvalue with the largest real part. The cumulant generating function after many periods $t \rightarrow \mathcal{N} \mathcal{T}, \mathcal{N} \gg 1$, becomes fully determined by the Floquet eigenvalue $\phi(\chi) \equiv \max _{i}\left[\lambda_{i}(\chi)\right]$ with the largest real-part:

$$
\left.\mathcal{S}(\chi, \mathcal{N} \mathcal{T})\right|_{\mathcal{N} \gg 1}=\left.\ln \sum_{i} e^{\lambda_{i}(\chi) \mathcal{N} \mathcal{T}}\left\langle 1 \mid p_{i}(\chi, \mathcal{N} \mathcal{T})\right\rangle\right|_{\mathcal{N} \gg 1} \simeq \mathcal{N} \mathcal{T} \phi(\chi)
$$

Now it is clear that the statistics of quantum pumps is encoded in the Floquet eigenvalue $\phi(\chi)$. We proceed with a discussion on two-parameter pumping and evaluate the cumulant generating function using the adiabatic expansion.

\subsection{Adiabatic pumping and classical Berry phase}

Two-parameter pumps are usually referred to as adiabatic pumps since they function accurately only in the low-frequency regime. Two-parameter pumps have three main stages of the cycle: loading the electron on the island by lowering the first barrier, capturing the electron on the island, and unloading or emitting the electron from the island by lowering the second barrier. To visualize a pumping cycle with two parameters, we assume electrostatically induced barriers and the role of the control parameters is played by the time-dependent gate voltages, Fig. 2.9 (a). Intuitively, we can guess that the three main stages of the two-parameter pumping cycle are realized successfully when we allow the system to reach the equilibrium charge state at each stage. An equilibration at the stages of the dynamic process automatically implies the adiabaticity and hints towards the adiabatic description of the two-parameter pumps. From the numeric simulation as shown in Fig. 2.9 (b), we observe frequency-independent 

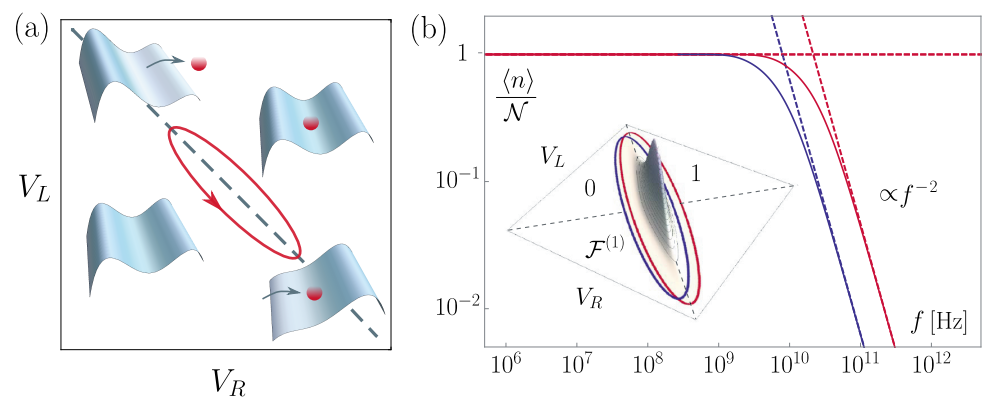

Figure 2.9. Two-parameter single-electron pumping. (a) Adiabatic pumping is achieved by slowly modulating both gate voltages periodically in time as illustrated by the red elliptic contour. (b) Pumped charge for the two-parameter pump. The driving protocol is shown together with the Berry curvature $\mathcal{F}^{(1)}$ in the inset, where the stable charge configuration of the island is also indicated ( 0 or 1 electrons). The solid lines are numerical results, while the dashed lines are results of the adiabatic and high-frequency approximations. The red line is obtained with driving parameters that maximize the breakdown frequency.

behavior of pumped charge in the low-frequency range. As we will see, pumping in the adiabatic regime is described by a geometric object, a classic analogue of the Berry curvature. Adiabatic theory allows for a geometric optimization of the driven devices and therefore is a crucial concept not only for two-parameter single-electron pumps, but any low dimensional nano-device driven by more than one independent parameter.

In the adiabatic expansion, we effectively treat the time-derivative $-\frac{d}{d t}$ in Eq. (2.5) as the perturbation. In practice, it is the time-derivative of the instantaneous eigenstate that has to be small in the adiabatic process. We expand the eigenvalue and eigenvector as $\phi(\chi)=\sum_{k=0}^{\infty} \phi^{(k)}(\chi)$ and $|p(\chi, t)\rangle=\sum_{k=0}^{\infty}\left|p^{(k)}(\chi, t)\right\rangle$ and collect terms of the same order. In the lowest order of the adiabatic expansion, when driving is infinitely slow, we find our system following the instantaneous state $\left|p^{(0)}(\chi, t)\right\rangle$ :

$$
\mathbf{L}(\chi, t)\left|p^{(0)}(\chi, t)\right\rangle=\lambda^{(0)}(\chi, t)\left|p^{(0)}(\chi, t)\right\rangle,
$$

where $\lambda^{(0)}(\chi, t)$ is the instantaneous eigenvalue of $\mathbf{L}(\chi, t)$ with the largest real-part and $\left|p^{(0)}(\chi, t)\right\rangle$ is the corresponding eigenvector. Now we can express the exact Floquet eigenvalue from Eq. (2.5) as a sum of the instantaneous value and higher order corrections:

$$
\phi(\chi)=\phi^{(0)}(\chi)-\int_{0}^{\mathcal{T}} \frac{d t}{\mathcal{T}}\left\langle p^{(0)}(\chi, t)\left|\frac{d}{d t}\right| p(\chi, t)\right\rangle,
$$

where $\phi^{(0)}(\chi)=\int_{0}^{\mathcal{T}} \frac{d t}{\mathcal{T}} \lambda^{(0)}(\chi, t)$ is the average of the instantaneous eigenvalue. To the first order, we find the classical analogue of the Berry phase: 
[18, 19, 20]

$$
\phi^{(1)}(\chi)=-\int_{0}^{\mathcal{T}} \frac{d t}{\mathcal{T}}\left\langle p^{(0)}(\chi, t)\left|\frac{d}{d t}\right| p^{(0)}(\chi, t)\right\rangle .
$$

With help of Stoke's theorem, we rewrite the classical Berry phase in order to demonstrate it's geometric nature:

$$
\phi^{(1)}(\chi)= \pm f \iint_{\mathcal{S}} d V_{L} d V_{R} \mathcal{F}(\chi, \mathbf{V}),
$$

where the sign is given by the orientation of the contour enclosing the surface $\mathcal{S}$ in the parameter space, and the object under the integral is a classical analogue of the Berry curvature:

$$
\mathcal{F}(\chi, \mathbf{V})=\left[-\partial_{V_{L}}, \partial_{V_{R}}\right] \cdot\left\langle p^{(0)}(\chi, \mathbf{V})|\nabla \mathbf{V}| p^{(0)}(\chi, \mathbf{V})\right\rangle .
$$

For a device controlled by a single parameter, the classical Berry curvature and therefore also the Berry phase vanishes. We proceed to the second order:

$$
\phi^{(2)}(\chi)=-\int_{0}^{\mathcal{T}} \frac{d t}{\mathcal{T}}\left\langle p^{(0)}(\chi, t)\left|\frac{d}{d t} \mathbf{R}(\chi, t) \frac{d}{d t}\right| p^{(0)}(\chi, t)\right\rangle
$$

having used $\left|p^{(1)}(\chi, t)\right\rangle=\mathbf{R}(\chi, t) \frac{d}{d t}\left|p^{(0)}(\chi, t)\right\rangle$ as in standard perturbation theory, where $\mathbf{R}(\chi, t)$ is the pseudo-inverse of $\mathbf{L}(\chi, t)-\lambda^{(0)}(\chi, t)$ [21]. Equation (2.7) is important as it allows us to evaluate the charge transfer statistics for single-parameter pumps to first non-trivial order in the driving frequency. The first two cumulants of the adiabatic cumulant generating function (2.6) and one order beyond (2.7) are evaluated and compared to exact numerical solutions in the Publication III. We move on to discuss single-parameter pumping and the high-frequency expansion of the cumulant generating function.

\subsection{Non-adiabatic pumping}

The cycle of a one-parameter pump is quite different from a two-parameter one. In the first stage of the cycle, we load an electron to the island when the left barrier is lowered. In the second stage, we quickly raise the barrier such that electron won't tunnel back and continue to raise the barrier until the electron is emitted out of the island. During the entire cycle the second barrier remains constant with non-zero amplitude, see Fig. 2.10 (a). The main difference here is that the pumping process has to be non-adiabatic in order to deliver a finite current [22]. When operated adiabatically, one-parameter pumps produce large noise, due to electrons tunneling in 

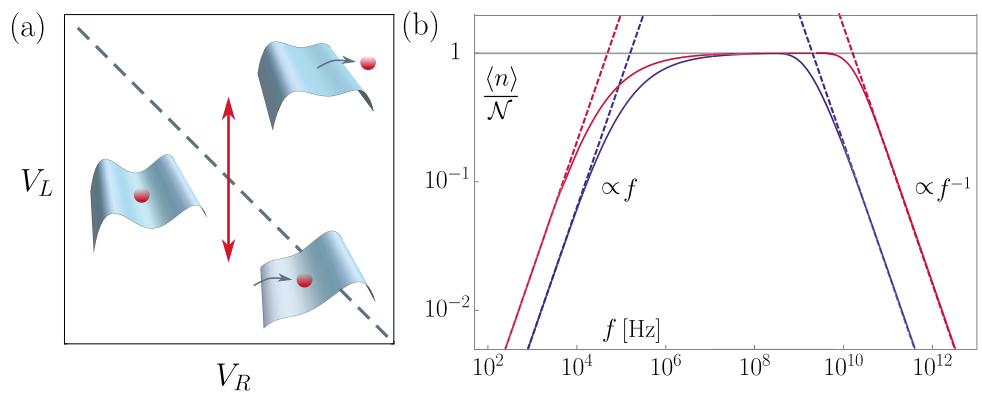

Figure 2.10. (a) Non-adiabatic charge pumping is achieved by modulating a single gate voltage periodically in time as illustrated by the red line. The insets show the gate-defined confining potential. (b) Average pumped charge per period as a function of the driving frequency. The solid lines are numerical results, while the dashed lines are results of the adiabatic and high-frequency expansions. The red line is obtained with driving parameters that maximize the breakdown frequency.

and out of the island, but zero current on average. As we approach the high-frequency regime, we hit a critical frequency value, at which the pumped current decreases and deviates strongly from the quantized value as shown in Fig. 2.10 (b). The ultimate goal is to pump as accurately and as fast as possible. We would like to shift the breakdown frequency towards the higher frequencies and realize accurate single-electron pumping at $\mathrm{GHz}$ frequencies. In order to access the high-frequency range, we use the Magnus-Floquet expansion [23, 24]. Here, instead of expanding the periodic state, we focus on the time-evolution operator in the Floquet form:

$$
\mathbf{U}(\chi, t)=\hat{T}\left\{e^{\int_{0}^{t} d t^{\prime} \mathbf{L}\left(\chi, t^{\prime}\right)}\right\}=u(\chi, t) e^{\mathbf{F}(\chi) t},
$$

where we identify the Floquet eigenvalue $\phi(\chi)$ as the eigenvalue of $\mathbf{F}(\chi)$ with the largest real-part. By inserting the time-evolution in the Floquet form back into the rate equation (2.1), we obtain the evolution equation for $u(\chi, t)$ :

$$
\frac{d}{d t} u(\chi, t)=\mathbf{L}(\chi, t) u(\chi, t)-u(\chi, t) \mathbf{F}(\chi) .
$$

According to the Magnus' proposal [25] regarding the linear evolution equations, we express the solution as the exponential of some function $\Lambda(\chi, t)$ as

$$
u(\chi, t)=e^{\Lambda(\chi, t)}
$$

Following the Floquet theorem, the exponential ansatz Eq. (2.10) also has to be time periodic such that $u(\chi, t)=u(\chi, t+\mathcal{T})$ and $\Lambda(\chi, t)=\Lambda(\chi, t+\mathcal{T})$. From Eq. (2.9), follows the time evolution equation

$$
\frac{d}{d t} e^{\Lambda(\chi, t)}=\mathbf{L}(\chi, t) e^{\Lambda(\chi, t)}-e^{\Lambda(\chi, t)} \mathbf{F}(\chi),
$$


which implies an iterative solution for $\Lambda(\chi, t)$ :

$$
\frac{d}{d t} \Lambda(\chi, t)=\sum_{k=0}^{\infty} \frac{B_{k}}{n !} \operatorname{ad}_{\Lambda(\chi, t)}^{k}\left(\mathbf{L}(\chi, t)+(-1)^{k+1} \mathbf{F}(\chi)\right),
$$

Here, $B_{k}$ stands for the Bernoulli numbers, and $\operatorname{ad}_{X}^{k}$ is a linear operator which acts according to

$$
\operatorname{ad}_{X}^{0} Y=Y, \quad \operatorname{ad}_{X} Y=[X, Y], \quad \operatorname{ad}_{X}^{k} Y=\left[X, \operatorname{ad}_{X}^{k-1} Y\right] .
$$

As a final step, we consider the expansions:

$$
\mathbf{F}(\chi)=\sum_{k=0}^{\infty} \mathbb{F}^{(k)}(\chi), \quad \Lambda(\chi, t)=\sum_{k=0}^{\infty} \Lambda^{(k)}(\chi, t),
$$

and arrive at the Magnus-Floquet expansion:

$$
\mathbf{U}(\chi, t)=\exp \left[\sum_{k=0}^{\infty} \Lambda^{(k)}(\chi, t)\right] \exp \left[t \sum_{k=0}^{\infty} \mathbb{F}^{(k)}(\chi)\right] .
$$

In this expansion, the terms $\mathbb{F}^{(k)}(\chi)$ can be determined independently at $t=\mathcal{T}$, when the time-evolution operator shrinks to $\mathbf{U}(\chi, \mathcal{T})=e^{\mathbf{F}(\chi) \mathcal{T}}$. The first term is simply the period average of the rate matrix:

$$
\mathbb{F}^{(0)}(\chi)=\int_{0}^{\mathcal{T}} \frac{d t}{\mathcal{T}} \mathbf{L}(\chi, t)
$$

Up until now, there are no signs of time-ordering, but as we move to the second term:

$$
\mathbb{F}^{(1)}(\chi)=\int_{0}^{\mathcal{T}} \frac{d t}{2} \int_{0}^{t} \frac{d t^{\prime}}{\mathcal{T}}\left[\mathbf{L}(\chi, t), \mathbf{L}\left(\chi, t^{\prime}\right)\right]
$$

we notice a commutator of the rate matrices at different times, which is an imprint of the time-ordering operation (2.8). The Floquet eigenvalues in the Magnus-Floquet expansion $\phi(\chi)=\sum_{k=0}^{\infty} \varphi^{(k)}(\chi)$ are eigenvalues of the corresponding terms in the expansion of the Floquet operator (2.11). The first term is the eigenvalue of the period averaged rate matrix:

$$
\varphi^{(0)}(\chi)\left|\mathbb{P}^{(0)}(\chi)\right\rangle=\mathbb{F}^{(0)}(\chi)\left|\mathbb{P}^{(0)}(\chi)\right\rangle,
$$

where $\left|\mathbb{P}^{(0)}(\chi)\right\rangle$ are the corresponding eigenvectors. The first term of the high-frequency expansion (2.12) represents the response of the system when the driving frequency exceeds all other inverse time scales of the system by more than one order. Under such conditions, the behavior of the system resembles a stationary process represented by the average rates. The higher order correction to the Floquet eigenvalue reads

$$
\varphi^{(1)}(\chi)=\left\langle\mathbb{P}^{(0)}(\chi)\left|\mathbb{F}^{(1)}(\chi)\right| \mathbb{P}^{(0)}(\chi)\right\rangle .
$$

We use both cumulant generating functions (2.12) and (2.13) to evaluate the first two cumulants and estimate the breakdown frequency of the one-parameter pump in Publication III. 


\section{Coherent single-electron transport}

This chapter is a brief overview of coherent transport and thermodynamics in periodically driven conductors. We begin with an introduction to the Floquet scattering matrix formalism and proceed with the formulation of non-equilibrium thermodynamics of coherent driven systems. At the end of this chapter, we focus on the adiabatic-linear response regime.

\subsection{Floquet scattering theory}

When the size of a conductor becomes comparable to the mean free path of the charge or heat carriers, it is referred to as coherent conductor [26]. Carrier dynamics in coherent conductors is described by quantum mechanics. When a particle from a classical thermal reservoir is injected in the coherent conductor, it behaves as a non-interacting excitation on top of the Fermi sea. This phenomenon can be accurately described by elastic scattering theory.

Time-dependent driving, for example, an oscillating confining potential allows us to manipulate and transfer single particles between the conductors. In this case, an incoming particle can absorb or emit discrete quanta of energy due to interactions with a dynamic scatterer. Inelastic processes are captured by a Floquet scattering theory [27, 28]. Elements of the Floquet scattering matrix $S_{E_{n}, E}^{\alpha \beta}$ depend on two energies: the energy of an incoming electron, $E$, and the energy of an outgoing electron after the interaction, $E_{n}=E+n \hbar \omega$. Floquet scattering amplitudes follow general rules, dictated by unitarity and time-reversal symmetry. First, the sum rules

$$
\sum_{n} \sum_{\alpha}\left|S_{E_{n}, E}^{\alpha \beta}\right|^{2}=\sum_{n} \sum_{\alpha}\left|S_{E, E_{n}}^{\beta \alpha}\right|^{2}=1
$$

which follow from the unitarity of the Floquet scattering matrix [27], ensure the conservation of probabilities in single-particle scattering events. 
The double sum runs over all terminals and all integers $n$, for which $E_{n}>0$. Second, the time-reversal symmetry of Schrödinger's equation implies:

$$
S_{E_{n}, E}^{\alpha \beta}=\mathrm{T}_{\mathbf{B}} \mathbf{T}_{\lambda} S_{E, E_{n}}^{\beta \alpha},
$$

where operators $T_{B}$ and $T_{\lambda}$ indicate the reversal of external magnetic fields and driving protocols, respectively.

The Floquet scattering matrix is essentially a collection of transmission and reflection amplitudes, which describe particle and energy transport from the lead $\beta$ to the lead $\alpha$. In practice, we have to solve the timedependent Schrödinger equation describing the evolution of an incoming plane wave $\left|\Psi_{E}^{\beta}(t)\right\rangle$ with fixed energy $E$ :

$$
i \hbar \partial_{t}\left|\Psi_{E}^{\beta}(t)\right\rangle=\left[\frac{p^{2}}{2 m}+V(x, t)\right]\left|\Psi_{E}^{\beta}(t)\right\rangle,
$$

with respect to the scattering boundary conditions

$$
\left\langle x_{\alpha} \mid \Psi_{E}^{\beta}(t)\right\rangle=\delta_{\alpha \beta} \xi(E) e^{i k(E) x_{\alpha}-i E t / \hbar}+\int_{0}^{\infty} d E^{\prime} S_{E^{\prime}, E}^{\alpha \beta} \xi\left(E^{\prime}\right) e^{i k\left(E^{\prime}\right) x_{\alpha}-i E^{\prime} t / \hbar} .
$$

Here, $m$ denotes the mass of the particle, $p$ the momentum operator, $x$ the position operator and $V(t)$ a periodically time-dependent scattering potential, whose range is limited to the central conductor. Inside the leads, the Hamiltonian reduces to $H_{0} \equiv p^{2} / 2 m$. In Eq. (3.3), we have used the abbreviation $k(E) \equiv \sqrt{2 m E / \hbar^{2}}$ for the wave number and introduced the normalization factor [29] $\xi(E) \equiv \sqrt{m / 2 \pi k(E) \hbar^{2}}$. When the external driving $V(t)=V(t+\mathcal{T})$ is periodic, the Floquet theorem implies that the solutions of the Schrödinger equation (3.2) are of the following form

$$
\left|\Psi_{\varepsilon}^{\beta}(t)\right\rangle=e^{-i \varepsilon t / \hbar}\left|\Phi_{\varepsilon}^{\beta}(t)\right\rangle,
$$

where Floquet scattering states $\left|\Phi_{\varepsilon}^{\beta}(t+\mathcal{T})\right\rangle=\left|\Phi_{\varepsilon}^{\beta}(t)\right\rangle$ are periodic in time. The energy $\varepsilon$ associated with Floquet states is defined only up to an integer number of energy quanta and is therefore referred to as quasienergy $\varepsilon^{\prime}=\varepsilon+n \hbar \omega$. For the solution in the Floquet form (3.4) to be compatible with (3.3) we identify $E$ with quasienergy:

$$
E^{\prime}-E \equiv n \hbar \omega, \quad \omega \equiv 2 \pi / \mathcal{T}
$$

where $n \in \mathbb{Z}$ is an arbitrary integer. On the formal level, the Floquet theorem serves as a proof of the statement that the plane wave traversing the conductor can exchange only discrete energy quanta of size $\hbar \omega$ with 
the external controller. The boundary conditions for the Floquet scattering states read

$$
\left\langle x_{\alpha} \mid \Phi_{E}^{\beta}(t)\right\rangle=\delta_{\alpha \beta} \xi(E) e^{i k(E) x_{\alpha}}+\sum_{n \in \mathbb{Z}} S_{E_{n}, E}^{\alpha \beta} \xi\left(E_{n}\right) e^{i k\left(E_{n}\right) x_{\alpha}-i n \omega t} .
$$

Here, we formally define the Floquet scattering amplitudes $S_{E_{n}, E}^{\alpha \beta}$ and replace $E^{\prime}$ with $E_{n}$, where $E_{n}=E+n \hbar \omega$. We proceed with formulating the mean particle and energy currents flowing from the reservoirs into the system.

First, the operators of local particle and energy density at the position $x_{\alpha}$ in the lead $\alpha$ are given by

$$
\begin{aligned}
& n_{\rho}\left(x_{\alpha}\right) \equiv \delta\left(x-x_{\alpha}\right), \\
& n_{E}\left(x_{\alpha}\right) \equiv \frac{1}{2}\left(n_{\rho}\left(x_{\alpha}\right) H_{0}+H_{0} n_{\rho}\left(x_{\alpha}\right)\right),
\end{aligned}
$$

where $H_{0}=p^{2} / 2 m$ denotes the Hamiltonian of a free particle. The corresponding local current operators are then determined by the continuity equations

$$
\begin{aligned}
& \partial_{t}\left\langle\psi(t)\left|n_{\rho}\left(x_{\alpha}\right)\right| \psi(t)\right\rangle=-\partial_{x_{\alpha}}\left\langle\psi(t)\left|J_{\rho}\left(x_{\alpha}\right)\right| \psi(t)\right\rangle, \\
& \partial_{t}\left\langle\psi(t)\left|n_{E}\left(x_{\alpha}\right)\right| \psi(t)\right\rangle=-\partial_{x_{\alpha}}\left\langle\psi(t)\left|J_{E}\left(x_{\alpha}\right)\right| \psi(t)\right\rangle .
\end{aligned}
$$

Here, $|\psi(t)\rangle$ denotes an arbitrary solution of the Schrödinger equation (3.2). From (3.6), we obtain

$$
\begin{aligned}
& J_{\rho}\left(x_{\alpha}\right)=\frac{1}{2 m}\left[p, \delta\left(x-x_{\alpha}\right)\right], \\
& J_{E}\left(x_{\alpha}\right)=\frac{1}{4 m}\left[H_{0},\left[p, \delta\left(x-x_{\alpha}\right)\right]\right],
\end{aligned}
$$

where square brackets indicate the usual commutator. Consequently, the cycle mean values of the single-particle currents with respect to the Floquet scattering states (3.5) become

$$
\begin{aligned}
& \left\langle J_{\rho}\left(x_{\alpha}\right)\right\rangle=\frac{1}{\mathcal{T}} \int_{0}^{\mathcal{T}} d t\left\langle\Phi_{E}^{\beta}(t)\left|J_{\rho}\left(x_{\alpha}\right)\right| \Phi_{E}^{\beta}(t)\right\rangle=\frac{1}{h}\left(\delta_{\beta \alpha}-\sum_{n \in \mathbb{Z}}\left|S_{E_{n}, E}^{\alpha \beta}\right|^{2}\right), \\
& \left\langle J_{E}\left(x_{\alpha}\right)\right\rangle=\frac{1}{\mathcal{T}} \int_{0}^{\mathcal{T}} d t\left\langle\Phi_{E}^{\beta}(t)\left|J_{E}\left(x_{\alpha}\right)\right| \Phi_{E}^{\beta}(t)\right\rangle=\frac{1}{h}\left(\delta_{\beta \alpha} E-\sum_{n \in \mathbb{Z}} E_{n}\left|S_{E_{n}, E}^{\alpha \beta}\right|^{2}\right) .
\end{aligned}
$$

Assuming non-interacting particles the thermodynamic currents can be obtained by averaging (3.7) with the Fermi distribution $f_{E}^{\beta}=1 / 1+\exp [(E-$ $\left.\left.\mu_{\beta}\right) / T_{\beta}\right]$ of the reservoir $\beta$ with Boltzmann's constant being set to 1 throughout. After summing over the contributions from all individual reservoirs, 


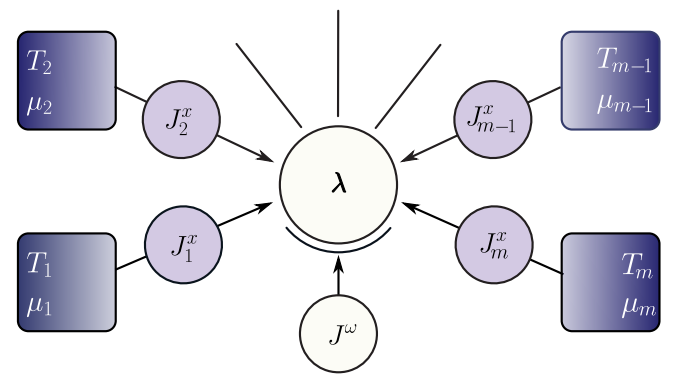

Figure 3.1. Dynamical multi-terminal conductor. A central scattering region subject to the periodic driving fields $\boldsymbol{\lambda}$ is connected to $m$ reservoirs with chemical potentials $\mu_{1}, \ldots, \mu_{m}$ and temperatures $T_{1}, \ldots, T_{m}$. Each reservoir injects a constant mean current of particles $(x=\rho)$ and heat $(x=q)$ into the conductor. Additionally, the external driving provides a continuous inflow of energy proportional to the photon flux $J^{\omega}$.

we obtain the microscopic expressions for the net particle and energy current flowing through the terminal $\alpha$ :

$$
\begin{aligned}
& J_{\alpha}^{\rho}=\frac{1}{h} \int_{0}^{\infty} d E \sum_{\beta}\left(\delta_{\beta \alpha}-\sum_{n}\left|S_{E_{n}, E}^{\alpha \beta}\right|^{2}\right) f_{E}^{\beta} \text { and } \\
& J_{\alpha}^{E}=\frac{1}{h} \int_{0}^{\infty} d E \sum_{\beta}\left(E \delta_{\beta \alpha}-\sum_{n} E_{n}\left|S_{E_{n}, E}^{\alpha \beta}\right|^{2}\right) f_{E}^{\beta} .
\end{aligned}
$$

\subsection{Thermodynamics of coherent driven conductors}

Particle and energy currents follow the conservation laws:

$$
\sum_{\alpha} J_{\alpha}^{\rho}=0 \quad \text { and } \quad \hbar \omega J^{\omega}+\sum_{\alpha} J_{\alpha}^{E}=0
$$

where $\hbar \omega J^{\omega}$ is the work done by external driving sources:

$$
\hbar \omega J^{\omega}=-\sum_{\alpha} J_{\alpha}^{E}=\frac{1}{h} \int_{0}^{\infty} d E \sum_{\alpha \beta} \sum_{n} n \hbar \omega\left|S_{E_{n}, E}^{\alpha \beta}\right|^{2} f_{E}^{\beta}
$$

Here, $J^{\omega}$ corresponds to the average current of energy quanta or photon flux injected into the conductor, see Fig. 3.1. Its physical interpretation as a photon flux relies on the fact that particles during the interaction with driving fields can exchange only discrete units of energy.

Before we continue, we make sure that the fluxes (3.8) and (3.9) follow the first and the second law of thermodynamics:

$$
\sum_{\alpha} J_{\alpha}^{q}+\hbar \omega J^{\omega}+\sum_{\alpha} \mu_{\alpha} J_{\alpha}^{\rho}=0 \quad \text { and } \quad \sigma \equiv-\sum_{\alpha} J_{\alpha}^{q} / T_{\alpha} \geq 0
$$

where $\sum_{\alpha} J_{\alpha}^{q}=\sum_{\alpha} J_{\alpha}^{E}-\mu_{\alpha} J_{\alpha}^{\rho}$ is the total heat flux, $\hbar \omega J^{\omega}$ is the work done by external sources, $\sum_{\alpha} \mu_{\alpha} J_{\alpha}^{\rho}$ is the average electrical power absorbed by the system, and $\sigma$ denotes the total rate of entropy production 
accompanying the transport process. In fact, the unitarity of the Floquet scattering matrix (3.1) ensures that mean fluxes obey the fundamental laws of thermodynamics.

It is convenient to introduce affinities with some reference chemical potential $\mu$ and temperature $T$ :

$$
F^{\omega} \equiv \hbar \omega / T, \quad F_{\alpha}^{\rho} \equiv\left(\mu_{\alpha}-\mu\right) / T, \quad F_{\alpha}^{q} \equiv 1 / T-1 / T_{\alpha},
$$

and rewrite the laws (3.10) in terms of thermodynamic fluxes and forces. The first law becomes

$$
\sum_{\alpha} J_{\alpha}^{q}+T J^{\omega} F^{\omega}+\sum_{\alpha} T J_{\alpha}^{\rho} F_{\alpha}^{\rho}=0
$$

and the second law takes a bilinear form:

$$
\sigma=J^{\omega} F^{\omega}+\sum_{\alpha} J_{\alpha}^{\rho} F_{\alpha}^{\rho}+J_{\alpha}^{q} F_{\alpha}^{q} \geq 0
$$

On the level of mean fluxes, the structure of the first and the second law resembles the irreversible thermodynamics of non-equilibrium steady states [30]. The thermodynamic properties of the systems out of equilibrium, either in a steady-state or in a periodic state, appear to be similar. But as we will see, only the latter one has a geometric interpretation in terms of the Berry curvature.

We proceed with a discussion on the adiabatic-linear regime, when voltage bias and temperature difference between reservoirs, as well as the energy quanta associated with the driving frequency $\hbar \omega$, are assumed to be the smallest energy scales in the system.

\subsection{Adiabatic-linear response and Berry phase}

Full Floquet scattering amplitudes are quite complicated objects and are rarely accessible as an exact solution. Here, we make use of the adiabatic approximation to show how it significantly simplifies the problem and yet remains accurate when describing two-parameter quantum pumps.

First, as a standard starting point of linear response, we assume that the voltage bias and the temperature gradient are small compared to the reference values:

$$
F_{\alpha}^{\rho} \ll \mu / T, \quad F_{\alpha}^{q} \ll 1 / T
$$


This assumption allows us to expand the Fermi-Dirac distribution:

$$
f_{E}^{\beta} \simeq f_{E}-\partial_{E} f_{E}\left(T F_{\beta}^{\rho}+(E+\mu) T F_{\beta}^{q}\right) \quad \text { with } \quad f_{E}=\left.f_{E}^{\beta}\right|_{F_{\beta}^{\rho}=0, F_{\beta}^{q}=0} .
$$

The second key assumption does not limit the driving amplitude like in the linear response but requires adiabaticity instead. While allowing for arbitrary large driving amplitudes, we compare the driving frequency $\omega$ to the inverse traverse time $\delta_{E} / \hbar$, also known as dwell time or Wigner time delay [31]:

$$
\omega \ll \delta_{E} / \hbar
$$

Over the energy scale $\delta_{E}$, instantaneous scattering amplitudes $\mathcal{S}_{E, \lambda}^{\alpha \beta}$ change significantly [27]. For example, in the transmission resonance regime [32], $\delta_{E}$ is given by the half-width of the resonance. Under the adiabatic condition (3.12), Floquet scattering amplitudes are given by the Fourier coefficients of the instantaneous scattering amplitudes and higher order corrections:

$S_{E_{n}, E}^{\alpha \beta} \simeq \frac{1}{\mathcal{T}} \int_{0}^{\mathcal{T}} d t \mathcal{S}_{E, \boldsymbol{\lambda}}^{\alpha \beta} e^{i n \omega t}+\left(n \hbar \omega \partial_{E} \mathcal{S}_{E, \boldsymbol{\lambda}}^{\alpha \beta}+\hbar \omega A_{E, t}^{\alpha \beta}\right) e^{i n \omega t}, \quad$ with $\quad \mathcal{T} \equiv 2 \pi / \omega$,

where $\mathcal{S}_{E, \lambda}^{\alpha \beta}$ are frozen scattering amplitudes - instantaneous solutions of the Schrödinger's equation, and anomalous scattering amplitudes $A_{E, t}^{\alpha \beta}$ ensure the unitarity of the Floquet scattering matrix after approximation.

As a result of the approximations (3.11) and (3.13), the thermodynamic fluxes (3.8) and (3.9) become linear functions of the corresponding affinities given by

$$
\begin{aligned}
& J_{\alpha}^{x}=L_{\alpha}^{x \omega} F^{\omega}+\sum_{\beta} \sum_{y} L_{\alpha \beta}^{x y} F_{\beta}^{y} \text { and } \\
& J^{\omega}=L^{\omega \omega} F^{\omega}+\sum_{\alpha} \sum_{x} L_{\alpha}^{\omega x} F_{\alpha}^{x} \text { with } x, y=\rho, q .
\end{aligned}
$$

The kinetic coefficients appearing in these relations are

$$
\begin{aligned}
& L_{\alpha \beta}^{x y}=\frac{T}{h} \int_{0}^{\infty} d E\left(-\partial_{E} f_{E}\right) \xi_{E}^{x} \xi_{E}^{y} \int_{0}^{\mathcal{T}} \frac{d t}{\mathcal{T}}\left(\delta_{\alpha \beta}-\left|\mathcal{S}_{E, \boldsymbol{\lambda}}^{\alpha \beta}\right|^{2}\right), \\
& L_{\alpha}^{x \omega}=\frac{T}{h} \int_{0}^{\infty} d E\left(-\partial_{E} f_{E}\right) \xi_{E}^{x} \xi^{\omega} \sum_{\beta} \operatorname{Im}\left[\int_{0}^{\mathcal{T}} \frac{d t}{\mathcal{T}} \mathcal{S}_{E, \boldsymbol{\lambda}}^{\alpha \beta} \cdot \partial_{t} \mathcal{S}_{E, \boldsymbol{\lambda}}^{\alpha \beta *}\right], \\
& L_{\alpha}^{\omega x}=\frac{T}{h} \int_{0}^{\infty} d E\left(-\partial_{E} f_{E}\right) \xi_{E}^{x} \xi^{\omega} \sum_{\beta} \operatorname{Im}\left[\int_{0}^{\mathcal{T}} \frac{d t}{\mathcal{T}} \partial_{t} \mathcal{S}_{E, \boldsymbol{\lambda}}^{\beta \alpha} \cdot \mathcal{S}_{E, \boldsymbol{\lambda}}^{\beta \alpha *}\right], \\
& L^{\omega \omega}=\frac{T}{h} \int_{0}^{\infty} d E\left(-\partial_{E} f_{E}\right)\left(\xi^{\omega}\right)^{2} \sum_{\alpha \beta} \int_{0}^{\mathcal{T}} \frac{d t}{\mathcal{T}}\left|\partial_{t} \mathcal{S}_{E, \boldsymbol{\lambda}}^{\alpha \beta}\right|^{2} / 2 \quad \text { with } \\
& \xi_{E}^{\rho} \equiv 1, \quad \xi_{E}^{q} \equiv E-\mu, \quad \xi^{\omega} \equiv 1 / \omega, \quad x, y=\rho, q .
\end{aligned}
$$

This result shows that, under adiabatic-linear response conditions, the thermodynamic fluxes depend only on the frozen scattering amplitudes 
$\mathcal{S}_{E, \boldsymbol{\lambda}}^{\alpha \beta}$, which are significantly easier accessible than the full Floquet scattering amplitudes entering the non-linear expressions (3.8) and (3.9). Another interesting aspect of this result is the geometric interpretation of the coefficients representing the pumping effect $L_{\alpha}^{x \omega}$ and $L_{\alpha}^{\omega x}$. In fact, the adiabatic transmission coefficients, which enter the expressions (3.14) for $L_{\alpha}^{x \omega}$ and $L_{\alpha}^{\omega x}$, have the same structure as Berry's geometric phase [33]. They can, therefore, be rewritten as

$$
\begin{aligned}
& \operatorname{Im}\left[\int_{0}^{\mathcal{T}} \frac{d t}{\mathcal{T}} \mathcal{S}_{E, \boldsymbol{\lambda}}^{\alpha \beta} \cdot \partial_{t} \mathcal{S}_{E, \boldsymbol{\lambda}}^{\alpha \beta *}\right]=-\operatorname{Im}\left[\int_{0}^{\mathcal{T}} \frac{d t}{\mathcal{T}} \partial_{t} \mathcal{S}_{E, \boldsymbol{\lambda}}^{\alpha \beta} \cdot \mathcal{S}_{E, \boldsymbol{\lambda}}^{\alpha \beta *}\right]=\frac{1}{\mathcal{T}} \oint_{\gamma} d \boldsymbol{\lambda} \cdot \mathcal{A}_{E, \boldsymbol{\lambda}}^{\alpha \beta}, \\
& \text { where } \mathcal{A}_{E, \boldsymbol{\lambda}}^{\alpha \beta} \equiv \operatorname{Im}\left[\mathcal{S}_{E, \boldsymbol{\lambda}}^{\alpha \beta} \nabla_{\boldsymbol{\lambda}} \mathcal{S}_{E, \boldsymbol{\lambda}}^{\alpha \beta *}\right]
\end{aligned}
$$

plays the role of a generalized vector potential known as the Berry connection, and $\gamma$ is the closed path in the space of control parameters that is encircled by the driving protocols. Via Stokes' theorem, this expression can be converted into a surface integral

$$
\operatorname{Im}\left[\int_{0}^{\mathcal{T}} \frac{d t}{\mathcal{T}} \mathcal{S}_{E, \boldsymbol{\lambda}}^{\alpha \beta} \partial_{t} \mathcal{S}_{E, \boldsymbol{\lambda}}^{\alpha \beta *}\right]=\frac{1}{\mathcal{T}} \int_{\Sigma_{\gamma}} d \mathbf{S} \cdot \boldsymbol{B}_{E, \boldsymbol{\lambda}}^{\alpha \beta} .
$$

The integral extends over an arbitrary surface $\Sigma_{\gamma}$ that is bounded by the curve $\gamma$ and $\mathcal{B}_{E, \lambda}^{\alpha \beta}$ denotes the Berry curvature. For a three-dimensional parameter space, this quantity is given by

$$
\mathcal{B}_{E, \boldsymbol{\lambda}}^{\alpha \beta}=\nabla_{\boldsymbol{\lambda}} \times \mathcal{A}_{E, \boldsymbol{\lambda}}^{\alpha \beta} .
$$

For the mean current in a two-parameter pump with two leads in the low temperature regime, we recover Brouwer's formula [34]:

$$
J^{\rho}=\omega / 4 \pi^{2} \iint d \lambda_{1} d \lambda_{2} \sum_{\beta} \mathcal{B}_{E}^{\alpha \beta}\left(\lambda_{1}, \lambda_{2}\right),
$$

where the Berry curvature explicitly reads as

$$
\sum_{\beta} \mathcal{B}_{E}^{\alpha \beta}\left(\lambda_{1}, \lambda_{2}\right)=\operatorname{Im}\left[\partial_{\lambda_{1}} S_{E, \boldsymbol{\lambda}}^{11 *} \partial_{\lambda_{2}} S_{E, \boldsymbol{\lambda}}^{11}+\partial_{\lambda_{2}} S_{E, \boldsymbol{\lambda}}^{12 *} \partial_{\lambda_{2}} S_{E, \boldsymbol{\lambda}}^{12}\right]
$$

Visualizing the Berry curvature in Fig. 3.2 in the control parameter space for a given system allows for an optimal driving protocol choice in order to maximize the pumped current and achieve quantization. The concept of the adiabatic approximation is crucial for nano-devices operated with more than one independent parameter, as it allows for geometric optimization via Berry curvature in the control parameter space.

This result shows that the adiabatic pumping effect in coherent conductors and in the Coulomb blockade regime is captured by the Berry phase. 


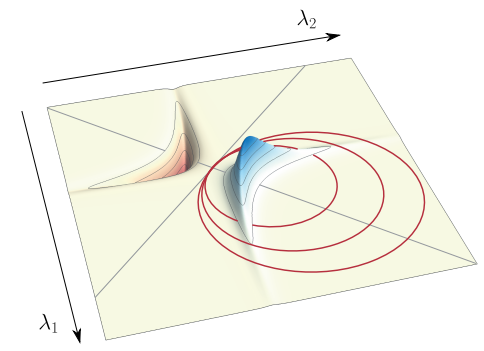

Figure 3.2. The Berry curvature for a tunable-barrier two-parameter single-electron pump. The grey lines indicate the two symmetries of this function. The circles correspond to the path encircled by the driving protocols. Specific scattering amplitudes and details of the system can be found in the Publication IV.

Current quantization in coherent conductors occurs in the transmission resonance regime [32]. The appearance of the transmission resonances is due to the interference of the incoming and outgoing plane waves where coherence is crucial. Single-electron pumping in the presence of the Coulomb blockade is incoherent and is dominated by classical physics, namely electrostatics. On the other hand, if we look at the broader picture behind pumping, we have two tunneling barriers with conductances $G_{1}(t)$ and $G_{2}(t)$. The adiabatic pumping cycle is realized by tuning the conductances via gate voltages $V_{1}(t)$ and $V_{2}(t)$ in a periodic manner to realize the loading and unloading stages of the cycle. If the details of a pumping device are not known, namely we are given a black box, there is certainly a unifying general idea behind adiabatic pumping in either coherent or incoherent systems. In Publication IV, we look at the adiabatic pumping from a thermodynamic point of view and raise the question of the thermodynamic cost. It turns out that during the optimal pumping cycle, one photon per cycle "is spent" to transfer one electron between two leads at zero temperature. The realistic, experimentally available tunable-barrier pumps are found to be thermodynamically more costly. The comparison between optimal quantum pumps [31] and tunable-barrier pumps is done using a power-flux trade-off relation:

$$
h\left(J^{\rho}\right)^{2} \leq \hbar \omega J^{\omega}
$$

which provides a lower bound on the mean work input $\hbar \omega J^{\omega}$ that is required to sustain a given pump current $J^{\rho}$. Derivation of the bound can be found in the Publication IV. 


\section{Summary of the findings}

In this thesis we have presented various techniques of quantum pump optimization. In Publication I, we optimize the regularity of single-electron emission events in a single-electron turnstile via distribution of electronic waiting times. In Publication II, by constructing the distribution of waiting times, we extract the in-and-out tunneling rates for a parasitic state strongly coupled to a superconducting charge detector. The hindering effect of charge traps can be mitigated when the time scales of charge fluctuations in a trap appear to be significantly different than those of the operation of the measured device. We combine full counting statistics with adiabatic and high-frequency expansions to optimize the breakdown frequency of two- and one-parameter pumps in Publication III. Maximizing the breakdown frequency is crucial in order to achieve the main metrological goal: the accurate and fast single-electron pumping. In the case of two-parameter pumps, we make use of Berry's adiabatic argument and maximize the mean current based on the symmetries of the Berry curvature. The same process of geometric optimization can be applied in the case of coherent quantum pumps, as we have shown in Publication IV. The adiabatic theory allows for pumping optimization in terms of the (input) work-(output) current relation. In Publication IV, we optimize quantum pumps with respect to thermodynamic quantities such as input work and entropy created during the pumping cycle. A term optimal pump, in this case, stands for a thermodynamically less costly pump. 
Summary of the findings 


\section{References}

[1] S. P. Giblin, M. Kataoka, J. D. Fletcher, P. See, T. J. B. M. Janssen, J. P. Griffiths, G. A. C. Jones, I. Farrer, and D. A. Ritchie. Towards a quantum representation of the ampere using single electron pumps. Nat. Commun., 3:930, 2012.

[2] J. P. Pekola, O.-P. Saira, V. F. Maisi, A. Kemppinen, M. Möttönen, Yu. A. Pashkin, and D. V. Averin. Single-electron current sources: Toward a refined definition of the ampere. Rev. Mod. Phys., 85:1472, 2013.

[3] F. Stein, D. Drung, L. Fricke, H. Scherer, F. Hohls, C. Leicht, M. Götz, C. Krause, R. Behr, E. Pesel, K. Pierz, U. Siegner, F. J. Ahlers, and H. W. Schumacher. Validation of a quantized-current source with $0.2 \mathrm{ppm}$ uncertainty. Appl. Phys. Lett., 107(10):103501, 2015.

[4] G. Yamahata, S. P. Giblin, M. Kataoka, T. Karasawa, and A. Fujiwara. Gigahertz single-electron pumping in silicon with an accuracy better than 9.2 parts in 107. Appl. Phys. Lett., 109(1):013101, 2016.

[5] G. Yamahata, S. P. Giblin, M. Kataoka, T. Karasawa, and A. Fujiwara. High-accuracy current generation in the nanoampere regime from a silicon single-trap electron pump. Sci. Rep., 7:45137, 2017.

[6] E. Bocquillon, V. Freulon, F. D. Parmentier, J.-M. Berroir, B. Plaçais, C. Wahl, J. Rech, T. Jonckheere, T. Martin, C. Grenier, D. Ferraro, P. Degiovanni, and G. Fève. Electron quantum optics in ballistic chiral conductors. Ann. Phys., 526(1-2):30, 2014.

[7] C. P. Heij, P. Hadley, and J. E. Mooij. Single-electron inverter. Appl. Phys. Lett., 78(8):1142, 2001.

[8] M. H. Devoret and R. J. Schoelkopf. Amplifying quantum signals with the single-electron transistor. Nature, 406:1039, 2000.

[9] X. Jehl, B. Voisin, T. Charron, P. Clapera, S. Ray, B. Roche, M. Sanquer, S. Djordjevic, L. Devoille, R. Wacquez, and M. Vinet. Hybrid MetalSemiconductor Electron Pump for Quantum Metrology. Phys. Rev. X, 3:021012, 2013.

[10] Y. V. Nazarov and Y. M. Blanter. Quantum Transport - Introduction to Nanoscience. Cambridge University Press, 1st edition, 2009.

[11] S. K. Gorman, Y. He, M. G. House, J. G. Keizer, D. Keith, L. Fricke, S. J. Hile, M. A. Broome, and M. Y. Simmons. Tunneling statistics for analysis of spin-readout fidelity. Phys. Rev. Appl., 8:034019, Sep 2017. 
[12] Yu. M. Galperin, Nanzhi Zou, and K. A. Chao. Resonant tunneling in the presence of a two-level fluctuator: Average transparency. Phys. Rev. B, 49:13728-13739, 1994.

[13] R. J. P. Keijsers, O. I. Shklyarevskii, and H. van Kempen. Point-Contact Study of Fast and Slow Two-Level Fluctuators in Metallic Glasses. Phys. Rev. Lett., 77:3411-3414, 1996.

[14] O. P. Balkashin, R. J. P. Keijsers, H. van Kempen, Yu. A. Kolesnichenko, and O. I. Shklyarevskii. Relaxation of two-level fluctuators in point contacts. Phys. Rev. B, 58:1294-1299, 1998.

[15] S. Oh, K. Cicak, J. S. Kline, M. A. Sillanpää, K. D. Osborn, J. D. Whittaker, R. W. Simmonds, and D. P. Pappas. Elimination of two level fluctuators in superconducting quantum bits by an epitaxial tunnel barrier. Phys. Rev. B, 74:100502, 2006.

[16] J. Schriefl, Y. Makhlin, A. Shnirman, and G. Schön. Decoherence from ensembles of two-level fluctuators. N. J. Phys., 8:1, 2006.

[17] N. M. Zimmerman, W. H. Huber, B. Simonds, E. Hourdakis, A. Fujiwara, Y.i Ono, Y. Takahashi, H. Inokawa, M. Furlan, and M. W. Keller. Why the longterm charge offset drift in Si single-electron tunneling transistors is much smaller (better) than in metal-based ones: Two-level fluctuator stability. $J$. Appl. Phys., 104(033710):033710, 2008.

[18] N. A. Sinitsyn and I. Nemenman. The Berry phase and the pump flux in stochastic chemical kinetics. Europhys. Lett., 77(5):58001, 2007.

[19] N. A. Sinitsyn and I. Nemenman. Universal Geometric Theory of Mesoscopic Stochastic Pumps and Reversible Ratchets. Phys. Rev. Lett., 99:220408, 2007.

[20] J. Ren, P. Hänggi, and B. Li. Berry-Phase-Induced Heat Pumping and Its Impact on the Fluctuation Theorem. Phys. Rev. Lett., 104:170601, 2010.

[21] C. Flindt, T. Novotný, A. Braggio, and A.-P. Jauho. Counting statistics of transport through Coulomb blockade nanostructures: High-order cumulants and non-Markovian effects. Phys. Rev. B, 82:155407, 2010.

[22] B. Kaestner and V. Kashcheyevs. Non-adiabatic quantized charge pumping with tunable-barrier quantum dots: a review of current progress. Rep. Prog. Phys., 78:103901, 2015.

[23] S. Blanes, F. Casas, J. A. Oteo, and J. Ros. The Magnus expansion and some of its applications. Phys. Rep., 470(5):151, 2009.

[24] M. Bukov, L. D’Alessio, and A. Polkovnikov. Universal high-frequency behavior of periodically driven systems: from dynamical stabilization to Floquet engineering. Adv. Phys., 64(2):139, 2015.

[25] W. Magnus. On the exponential solution of differential equations for a linear operator. Comm. Pure Appl. Math., 7(4):649, 1954.

[26] Y. Imry. Introduction to Mesoscopic Physics. Oxford University Press, 2nd edition, 2002.

[27] M. Moskalets and M. Büttiker. Floquet scattering theory of quantum pumps. Phys. Rev. B, 66:205320, 2002. 
[28] M. V. Moskalets. Scattering Matrix Approach to Quantum Transport. Imperial College Press, London, first edition, 2012.

[29] H. U. Baranger and A. D. Stone. Electrical linear-response theory in an arbitrary magnetic field: A new Fermi-surface formation. Phys. Rev. B, 40:8169, 1989.

[30] U. Seifert. Stochastic thermodynamics, fluctuation theorems and molecular machines. Rep. Prog. Phys., 75:126001, 2012.

[31] J. E. Avron, A. Elgart, G. M. Graf, and L. Sadun. Optimal Quantum Pumps. Phys. Rev. Lett., 87:236601, 2001.

[32] Y. Levinson, O. Entin-Wohlman, and P. Wölfle. Pumping at resonant transmission and transferred charge quantization. Physica A, 302(1):335, 2001.

[33] M. V. Berry. Quantal phase factors accompanying adiabatic changes. Proc. R. Soc. Lond. A, 392:45, 1984.

[34] P. W. Brouwer. Scattering approach to parametric pumping. Phys. Rev. B, 58:10135(R), 1998. 
Physics is a social science, collaboration is the key to success! 